\title{
Pharmacological protection of reperfusion injury in ST-segment elevation myocardial infarction. Gone with the wind?
}

\author{
Elisabetta Tonet ${ }^{1}$, Davide Bernucci ${ }^{1}$, Giampaolo Morciano ${ }^{2,3}$, Gianluca Campo ${ }^{1,3}$ \\ ${ }^{1}$ Cardiology Unit, Azienda Ospedaliera Universitaria di Ferrara, Cona (FE), Italy \\ ${ }^{2}$ Department of Morphology, Surgery and Experimental Medicine, Section of Pathology, Oncology and Experimental Biology, \\ University of Ferrara, Ferrara, Italy \\ ${ }^{3}$ Maria Cecilia Hospital, GVM Care \& Research, E.S: Health Science Foundation, Cotignola, Italy
}

Adv Interv Cardiol 2018; 14, 1 (51): 5-8

DOI: https://doi.org/10.5114/aic.2018.74349

Primary percutaneous coronary intervention $(\mathrm{PCI})$ represents the greatest progress in the treatment of ST-elevation myocardial infarction (STEMI) over the last 30 years. It reduces infarct size with an early restoration of blood flow within an ischemic myocardium. However, it is associated with a still partly mysterious paradox: reperfusion injury [1]. It is characterized by reversible mechanical dysfunction called "myocardial stunning" and microvascular obstruction or the "no reflow phenomenon" which corresponds to the presence of capillary damage, endothelial cell swelling, intraluminal thrombosis and injured cardiomyocytes. Several mediators are involved in ischemia/reperfusion injury; on one hand prolonged ischemia causes $\mathrm{pH}$ alterations, activation of anaerobic metabolism, dysfunction of ATPase-dependent ion transport mechanisms, intracellular calcium overload, cell swelling and finally cell death. On the other hand, after reperfusion, there is an increase of reactive oxygen species and tissue infiltration of neutrophils and pro-inflammatory cytokines with paradoxical exacerbation of the ischemic injury. Consequently, at the clinical level, reperfusion injury is associated with residual large infarct size, impaired left ventricular ejection fraction, ventricular arrhythmias and poor prognosis [1]. Figure 1 shows infarct size and its changes with reperfusion and prevention of reperfusion injury.

Therefore, combating reperfusion injury remains one of the most interesting and challenging frontiers in STEMI management. With the aim of mitigating the risk of ischemic-reperfusion injury in this setting several strategies have been proposed. Table I shows the major recent studies investigating reperfusion injury. Several studies have analyzed ischemic post-conditioning (IPoC) in animal models, with very promising results. It is a technique of cardiac protection in which repeated brief interruptions of blood flow are performed before restoring final myocardial reperfusion, improving myocardial metabolic recovery [1, 2]. On the wave of these pre-clinical findings, the largest IPoC trial in STEMI patients so far, the recent Third Danish Study of Optimal Acute Treatment of Patients With ST-elevation Myocardial Infarction-Ischemic Postconditioning (DANAMI3-iPOST), failed to demonstrate that routine $I P O C$ reduces the composite endpoint of all-cause death and hospitalization for heart failure at a median follow-up of about 3 years [2]. These results have been confirmed by a recent meta-analysis of randomized controlled trials investigating the usefulness of IPOC in STEMI patients undergoing primary $\mathrm{PCl}$ [3]. Other studies focused on some cytoprotective agents such as delcasertib: it is an antagonist of an isoenzyme of protein kinase C (PKC) involved in myocardial cell damage following ischemia. Experimental models demonstrated that delcasertib, selectively inhibiting delta PKC isoform, reduces infarct size and improves microvascular function [4]. However, a phase II randomized trial, the PROTECTION AMI trial, underlined the absence of significant reductions in myocardial biomarkers and ST segment indices and left ventricular ejection fraction in STEMI patients 3 months after the primary $\mathrm{PCl}$ so that the authors deduced that no benefit was evident in terms of death and re-hospitalizations [5]. So reperfusion injury appears to be a jigsaw puzzle in which the piece that combines pre-clinical models and clinical results is missing.

A possible keystone is represented by mitochondrial function. In particular, experimental evidence indicates

\section{Corresponding author:}

Gianluca Campo MD, Cardiovascular Unit, Azienda Ospedaliera Universitaria di Ferrara, Via Aldo Moro 8, 44124, Cona (FE), Italy, phone: +39 (0) 532236450, e-mail: cmpglc@unife.it

Received: 23.01.2018, accepted: 23.01.2018. 


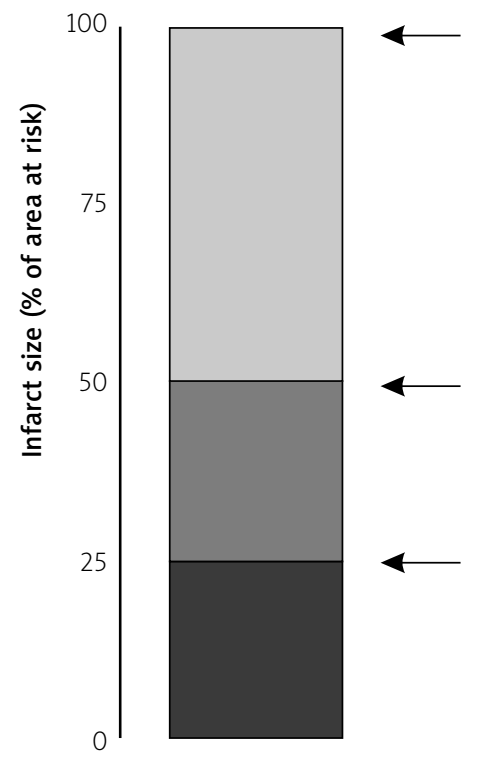

Myocardial ischemia in absence of reperfusion (no myocardial salvage)

Myocardial ischemia with timely reperfusion (50\% myocardial salvage)

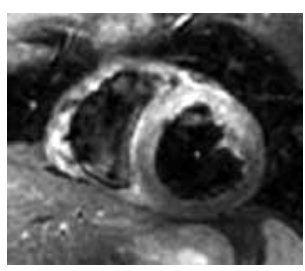

Myocardial ischemia with timely reperfusion and prevention of reperfusion injury (75\% myocardial salvage)

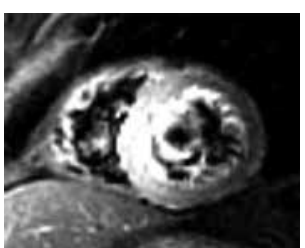

Figure 1. Infarct size and its reduction by timely reperfusion and prevention of reperfusion injury. Green: Myocardium salvaged by reperfusion. About half of the residual infarct size is due to reperfusion injury and it is preventable. Red: Myocardium salvaged by prevention of reperfusion injury. Benefits of timely reperfusion and prevention of reperfusion injury. Blue: Residual infarct size

Table I. Setting and limitations of recent major studies about reperfusion injury

\begin{tabular}{|c|c|c|c|}
\hline $\begin{array}{l}\text { Most important } \\
\text { studies }\end{array}$ & Pre-clinical concept & Clinical setting & $\begin{array}{c}\text { Major limitations in clinical } \\
\text { setting }\end{array}$ \\
\hline DANAMI-3-iPOST & Ischemic post-conditioning & $\begin{array}{l}\text { Failed to demonstrate that routine IPoC reduces } \\
\text { the composite endpoint of all-cause death and } \\
\text { hospitalization for heart failure at a median } \\
\text { follow-up of about } 3 \text { years }\end{array}$ & Culprit lesion \\
\hline PROTECTION AMI & $\begin{array}{c}\text { Delcasertib: inhibition of delta PKC } \\
\text { isoform }\end{array}$ & $\begin{array}{l}\text { Absence of significant reductions in myocardial } \\
\text { biomarkers and ST-segment indices and } \\
\text { LV ejection fraction in STEMI patients } 3 \text { months } \\
\text { after the primary PCI }\end{array}$ & $\begin{array}{l}\text { Time } \\
\text { Culprit lesion }\end{array}$ \\
\hline Ishii et al. & $\begin{array}{l}\text { Nicorandil: mitochondrial ATP-sensi- } \\
\text { tive potassium channel opener }\end{array}$ & $\begin{array}{l}\text { Uncertain benefit for fatal and non-fatal } \\
\text { outcomes }\end{array}$ & $\begin{array}{l}\text { Low statistical power } \\
\text { Selection bias } \\
\text { Small sample size }\end{array}$ \\
\hline MITOCARE & mPTP opening inhibitor (TRO40303) & No reduction of infarct size & $\begin{array}{l}\text { Time } \\
\text { Culprit lesion }\end{array}$ \\
\hline CIRCUS & $\begin{array}{c}\text { Cyclosporine A: keeping mPTP } \\
\text { closed }\end{array}$ & $\begin{array}{l}\text { No cardioprotective effects on myocardial } \\
\text { infarction severity }\end{array}$ & $\begin{array}{l}\text { Time } \\
\text { Culprit lesion }\end{array}$ \\
\hline EMBRACE STEMI & $\begin{array}{l}\text { Elamipretide: reduction of ROS } \\
\text { production }\end{array}$ & $\begin{array}{l}\text { No significant reduction of myocardial } \\
\text { infarct size }\end{array}$ & $\begin{array}{l}\text { Reduced primary analysis } \\
\text { population }\end{array}$ \\
\hline Campo et al. & mPTP C subunit & $\begin{array}{l}\text { High C subunit levels are related to lack of } \\
\text { ST-segment resolution and development of } \\
\text { a poor echocardiographic pattern }\end{array}$ & $\begin{array}{l}\text { Low statistical power for } \\
\text { clinical endpoints }\end{array}$ \\
\hline NACIAM & $\begin{array}{c}\text { Combined therapy: } \mathrm{N} \text {-acetylcysteine } \\
\text { and nitroglycerin }\end{array}$ & $\begin{array}{l}\text { No benefit for LV function and clinical } \\
\text { outcomes }\end{array}$ & $\begin{array}{l}\text { Uncertain mechanism of } \\
\text { action }\end{array}$ \\
\hline
\end{tabular}

$I P O C$ - ischemic post-conditioning, LV - left ventricular, $m P T P$ - mitochondrial permeability transition pore, PCI-percutaneous coronary intervention, PKC - protein kinase C, ROS - reactive oxygen species, STEMI-ST-elevation myocardial infarction.

that cardiomyocytes suffer and die from reperfusion injury because of the mitochondrial permeability transition pore (mPTP) opening at the time of reperfusion: physiological $\mathrm{pH}$ restoration, oxidative stress and calcium overload determine mPTP opening, allowing the passage of solutes into mitochondrial matrix, which is related to
ATP depletion, impaired metabolism, osmotic swelling and, when mitochondrial damage becomes irreversible, cell apoptosis $[1,6]$. Several studies have analyzed a mitochondrial ATP-sensitive potassium channel opener, nicorandil, demonstrating that it attenuates lethal reperfusion injury. Ishii et al. observed a beneficial effect of 
administration of nicorandil at the time of primary $\mathrm{PCl}$ in terms of early and later clinical events [7]. Nevertheless, recent studies about its influence at reperfusion time administration on fatal and non-fatal outcomes of STEMI patients showed the absence of any potential benefit [8]. Probably the major limitations of the study by Ishii et al. were related to the small sample size, selection bias and low statistical power.

The MITOCARE study evaluated the mitochondria-targeted and oxidative stress-dependent mechanism of TRO40303 compound but failed to demonstrate its efficacy in reducing infarct size of STEMI patients [9]. With the pre-clinical background of beneficial effects of cyclosporine A on keeping MPTP closed, the CIRCUS clinical trial failed to demonstrate its cardioprotective effects on myocardial infarction severity [10]. The major limitation that affected these studies on mitochondrial function and others, such as the above-mentioned PROTECTION AMI, was probably related to the long time between symptoms onset and cardioprotective agents' administration. Indeed, time can be considered the major determinant of successful reperfusion therapy: acting on reperfusion injury $6 \mathrm{~h}$ or more after symptoms onset means having to deal with necrotic cardiomyocytes and thus, to try a useless cardioprotective attempt. In addition, those studies suffer from another limitation related to the variability of the culprit lesion and infarct area of patients; in fact, one of the best conditions to study the mPTP opening mechanism seems to be the presence of left anterior descending occlusion with no evidence of collateral flow $[6,11]$.

Other studies have focused on elamipretide, a peptide showing a reduction of reactive oxygen species in experimental models by interacting with cardiolipin of the mitochondrial membrane [11]. Also in this case, experimental results did not correspond to clinical results: the EMBRACE STEMI study, avoiding the limitations of the trials described above in terms of time and culprit lesion, evaluated the administration of elamipretide in STEMI patients but failed to demonstrate a reduction of myocardial infarct size [11]. Nevertheless, mPTP could really represent the final crucial step of reperfusion injury. Promising results have been obtained analyzing MPTP structure and in particular the $C$ subunit, which appears to be the real pore component of the MPTP. But its additional value goes beyond preclinical results and cell cultures; indeed it was found to be an independent predictor of surrogate endpoints of myocardial reperfusion such as ST-segment resolution; patients with elevated serum levels of $C$ subunit were demonstrated to have no ST-segment resolution and developed a poor echocardiographic pattern 6 months after STEMI, with a close relationship with the occurrence of death and heart failure [12]. Further studies are needed to confirm these preliminary results.
A recent meta-analysis included data from randomized clinical trials by comparing drugs targeting mitochondrial function with the aim of evaluating any benefits for the hard endpoint in STEMI patients undergoing $\mathrm{PCl}$; the authors concluded that there was no benefit from administration of drugs targeting highly selective mitochondrial pathways, while drugs with a broad-spectrum mechanism of action reduced all clinical endpoints such as all-cause mortality and cardiovascular mortality [13].

Data obtained in all those studies demonstrated some important things: 1) the complex mechanism underlying reperfusion injury is still partly unknown; 2) this complexity is highlighted by the recurrent discrepancy between preclinical and clinical data; 3) however, clinical trials cannot be separated from cell culture analyses; 4) the complexity of reperfusion injury and the multiple facets that underlie it show that it is not possible to consider a single responsible mechanism, but rather multiple molecular interactions should be the object of future studies and targeted therapies. The recent NACIAM study by Pasupathy et al. demonstrated the potential usefulness of a combined therapy including $\mathrm{N}$-acetylcysteine and nitroglycerin in patients with STEMI treated with primary $\mathrm{PCl}$, with some limitations related to the effect on LV remodeling and clinical outcomes. The authors underlined the uncertainty as regards the exact mechanism of $\mathrm{N}$-acetylcysteine benefit; however, it seems that $\mathrm{N}$-acetylcysteine reduces mitochondrial alterations, the final step of reperfusion injury [14]. The NACIAM study represents the first step towards a combined therapy for cardioprotection. Another still emerging combination therapy includes exosomes and mesenchymal stem cells, which also seems to protect from reperfusion injury involving mitochondrial function, but the results are still uncertain [15]. So, further research should focus on a combination of molecules or on broad-spectrum molecules acting on MPTP and mitochondrial function that could be administered in STEMI patients as soon as possible after symptoms onset.

Early primary $\mathrm{PCl}$ has reduced short-term mortality by around 30\% compared to thrombolysis, but STEMI patients suffer from a residual hazard related to reperfusion injury that determines up to $50 \%$ of the final infarct size [1]. If pharmacological protection against reperfusion injury goes away with the wind, the mortality rate in patients with STEMI will still remain substantial. With the extensive preclinical background, future studies should concentrate on patient-oriented clinical endpoints for clinical decision-making.

\section{Conflict of interest}

The authors declare no conflict of interest.

\section{References}

1. Morciano G, Giorgi C, Bonora M, et al. Molecular identity of the mitochondrial permeability transition pore and its role in ischemia-reperfusion injury. J Mol Cell Cardiol 2015; 78: 142-53. 
2. Engstrom T, Kelbaek H, Helqvist S, et al. Effect of ischemic post-conditioning during primary percutaneous coronary intervention for patients with ST-segment elevation myocardial infarction: a randomized clinical trial. JAMA Cardiol 2017; 2: 490-7.

3. Mentias A, Mahmoud AN, Elgendy IY, et al. Ischemic post-conditioning during primary percutaneous coronary intervention: a meta-analysis of randomized trials. Catheter Cardiovasc Interv 2017; 90: 1059-67.

4. Inagaki F, Chen L, Ikeno F, et al. Inhibition of delta-protein kinase C protects against reperfusion injury of the ischemic heart in vivo. Circulation 2003; 108: 2304-7.

5. Lincoff AM, Roe M, Aylward P, et al. Inhibition of delta-protein kinase $C$ by delcasertib as an adjunct to primary percutaneous coronary intervention for acute anterior ST-segment elevation myocardial infarction: results of the PROTECTION AMI randomized controlled trial. Eur Heart J 2014; 35: 2516-23.

6. Bonora M, Morganti C, Morciano G, et al. Mitochondrial permeability transition involves dissociation of F1FO ATP synthase dimers and C-ring conformation. EMBO Rep 2017; 18: 1077-89.

7. Ishii $\mathrm{H}$, Ichimiya $\mathrm{S}$, Kanashiro $\mathrm{M}$, et al. Impact of a single intravenous administration of nicorandil before reperfusion in patients with ST-segment-elevation myocardial infarction. Circulation 2005; 112: 1284-8.

8. Campo G, Pavasini R, Morciano G, et al. Data on administration of cyclosporine, nicorandil, metoprolol on reperfusion related outcomes in ST-segment elevation myocardial infarction treated with percutaneous coronary intervention. Data in Brief 2017; 14: 197-205.

9. Atar D, Arheden H, Berdeaux A, et al. Effect of intravenous TRO40303 as an adjunct to primary percutaneous coronary intervention for acute ST-elevation myocardial infarction: MITOCARE study results. Eur Heart I 2015; 36: 112-9.

10. Cung TT, Morel O, Cayla G, et al. Cyclosporine before $\mathrm{PCl}$ in patients with acute myocardial infarction. N Engl J Med 2015; 373: 1021-31.

11. Gibson CM, Giugliano RP, Kloner RA, et al. EMBRACE STEM study: a phase $2 a$ trial to evaluate the safety, tolerability, and efficacy of intravenous MTP-131 on reperfusion injury in patients undergoing primary percutaneous coronary intervention. Eur Heart J 2016; 37: 1296-303.

12. Campo G, Morciano G, Pavasini R, et al. Fo ATP synthase C subunit serum levels in patients with ST-segment elevation myocardial infarction: preliminary findings. Int J Cardiol 2016; 221: 993-7.

13. Campo G, Pavasini R, Morciano G, et al. Clinical benefit of drugs targeting mitochondrial function as an adjunct to reperfusion in ST-segment elevation myocardial infarction: a meta-analysis of randomized clinical trials. Int J Cardiol 2017; 244: 59-66.

14. Pasupathy S, Tavella R, Grover S, et al. Early use of N-acetylcysteine (NAC) with nitrate therapy in patients undergoing primary percutaneous coronary intervention for ST-segment elevation myocardial infarction reduces myocardial infarct size (the NACIAM trial). Circulation 2017; 136: 894-903.

15. Arslan F, Lai RC, Smeets MB, et al. Mesenchymal stem cell-derived exosomes increase ATP levels, decrease oxidative stress and activate PI3K/Akt pathway to enhance myocardial viability and prevent adverse remodeling after myocardial ischemia/ reperfusion injury. Stem Cell Research 2013; 10: 301-12. 